\title{
Analytic solutions of the nonlinear radiation diffusion equation with an instantaneous point source in non-homogeneous media
}

\author{
Menahem Krief \\ Racah Institute of Physics, The Hebrew University, 9190401 Jerusalem, Israel
}

\begin{abstract}
Analytic solutions to the nonlinear radiation diffusion equation with an instantaneous point source for a non-homogeneous medium with a power law spatial density profile, are presented. The solutions are a generalization of the well known solutions for a homogeneous medium. It is shown that the solutions take various qualitatively different forms according to the value of the spatial exponent. These different forms are studied in detail for linear and non linear heat conduction. In addition, by inspecting the generalized solutions, we show that there exist values of the spatial exponent such the conduction front has constant speed or even accelerates. Finally, the various solution forms are compared in detail to numerical simulations, and a good agreement is achieved.
\end{abstract}

\section{INTRODUCTION}

Radiative heat waves are an important phenomena in many astrophysical and laboratory high energy density plasmas $[1-8]$. As a result, analytic solutions for the radiative heat equation play a key role in the analysis and design of high energy density experiments [2, 8, 11] and in the process of verification and validation of computer simulations $12+24$.

Analytic solutions for radiative heat waves with externally applied boundary conditions were developed in the seminal work of Marshak [25] which was further generalized in Refs. [26 33. Analytical solutions to the nonlinear diffusion equation with an instantaneous point source for a homogeneous medium was developed in the seminal works of Zel'dovich et al. [34 36] and Pattle 37. A solution for the linear diffusion equation in a non-homogeneous medium was developed in Ref. [38, in order to describe diffusion on fractal objects.

In this work we extend the solution of Zel'dovich et al. 34 36 and Pattle [37, and develop analytic solutions to the nonlinear radiation diffusion equation with an instantaneous point source for a non-homogeneous medium with a power law spatial density profile of the form $\rho_{0} r^{-\omega}$. Such profiles are widely used, for example, in modeling the interior and atmospheres of stars [39] 43 and galaxies 44-46. These solutions are analyzed in detail for both linear and non linear conduction. Different solution forms are examined for various ranges of the spatial exponent $\omega$. Finally, the solutions are compared with numerous numerical simulations.

\section{STATEMENT OF THE PROBLEM}

In situations where radiation heat conduction dominates and hydrodynamic motion is negligible, the material density is constant in time, and the heat flow is supersonic. A comparison between the dynamics of radiation conduction and compressible flow in the context of

\footnotetext{
* menahem.krief@mail.huji.ac.il
}

an instantaneous point source, also known as the strong explosion problem [12, 47 51 , will be detailed below in Sec. IIIB Assuming a constant spatial density, the radiation diffusion equation in one dimensional symmetry is given by:

$$
\frac{\partial u}{\partial t}=-\frac{1}{r^{d-1}} \frac{\partial}{\partial r}\left(r^{d-1} F\right)
$$

where $u(r, t)$ is the total energy per unit volume and $d=$ 1,2,3 for planar, cylindrical and spherical symmetries, respectively. The radiation energy flux obeys a Fick law:

$$
F=-D \frac{\partial}{\partial r}\left(a T^{4}\right),
$$

with $T$ the material temperature, $a$ the radiation constant, and the radiation diffusion coefficient:

$$
D=\frac{c}{3 \kappa_{R} \rho},
$$

where $c$ is the speed of light, $\kappa_{R}$ is the Rosseland mean opacity and $\rho$ is the material mass density. The flux $F$ is, in general, a non-linear function of $u$ and its derivative.

In this work, we assume power law opacity and energy equation of state, in the common form [8, 28, 31, 32, 52]:

$$
\frac{1}{\kappa_{R}(T, \rho)}=g T^{\alpha} \rho^{-\lambda}
$$

$$
u(T, \rho)=f T^{\beta} \rho^{1-\mu} .
$$

and an inhomogeneous density profile in a spatial power law form:

$$
\rho(r)=\rho_{0} r^{-\omega} .
$$

We note that in order for this density profile to contain a finite total mass, one must have $\omega<d$. In addition, it is evident from equations (3)-(6) that the above power 
laws are equivalent to a single temperature and spatial power law for the diffusion coefficient:

$$
D(r, T)=D_{0} r^{-\omega^{\prime}} T^{\alpha} .
$$

with $D_{0}=\frac{g c}{3 \rho_{0}^{\lambda+1}}, \omega^{\prime}=\omega(\lambda+1)$. The radiation diffusion coefficient as given in Eq. (3), is a non-linear function of $u$, in terms of $T$.

We consider an instantaneous point source, so that the initial energy density profile is given by:

$$
u(r, t=0)=Q \delta(\boldsymbol{r}),
$$

where $Q$ is the total initial energy, which is of course, constant in time for an infinite system:

$$
\int_{0}^{\infty} u(r, t) \mathcal{A}_{d} r^{d-1} d r=Q
$$

where the areal coefficient is:

$$
\mathcal{A}_{d}= \begin{cases}1 & d=1 \\ 2 \pi & d=2 \\ 4 \pi & d=3\end{cases}
$$

Using equations (2)-(6), the diffusion equation (1) takes the form:

$$
\frac{\partial u}{\partial t}=\frac{1}{r^{d-1}} \frac{\partial}{\partial r}\left(A r^{k+d-1}\left(r^{m} u\right)^{n} \frac{\partial}{\partial r}\left(r^{m} u\right)\right)
$$

where:

$$
\begin{gathered}
n=\frac{4+\alpha-\beta}{\beta}, \\
k=\omega(1+\lambda), \\
m=\omega(1-\mu), \\
A=\frac{16 \sigma g}{3 \beta f^{\frac{4+\alpha}{\beta}} \rho_{0}^{\lambda+1+\frac{(1-\mu)(\alpha+4)}{\beta}} .} .
\end{gathered}
$$

By defining the auxiliary variable:

$$
w(r, t)=r^{m} u(r, t),
$$

Eq. (11) takes the simple from:

$$
\frac{\partial w}{\partial t}=A r^{m-d+1} \frac{\partial}{\partial r}\left(r^{k+d-1} w^{n} \frac{\partial w}{\partial r}\right),
$$

It is seen from Eq. (11) that the energy flux can be written in terms of $w$ as:

$$
F(r, t)=-A r^{k} w^{n} \frac{\partial w}{\partial r} .
$$

The energy conservation law in Eq. (9), written in terms of $w$, reads:

$$
\int_{0}^{\infty} w(r, t) \mathcal{A}_{d} r^{d-m-1} d r=Q .
$$

In addition, the requirement to have vanishing energy density at infinity is:

$$
\left[r^{-m} w\right]_{r \rightarrow \infty}=0
$$

and from symmetry considerations, the energy current at the origin must vanish as well:

$$
\left[r^{k+d-1} w^{n} \frac{\partial w}{\partial r}\right]_{r \rightarrow 0}=0 .
$$

In this work, the solutions of Eq. 117) under the initial and boundary conditions $(19)-(21)$ will be studied in detail. We note that for $m=0$, Eq. (17) represents the general non-linear diffusion equation with an inhomogeneous diffusion coefficient of the form $D=A r^{k} w^{n}$, which is applicable to any other application of the diffusion equation (i.e. Ref. [38, which deals with linear diffusion in inhomogeneous media). In contrast, for radiation diffusion, due to the nonlinearity of the specific energy in terms of temperature, one has in general $m \neq 0$.

\section{SELF-SIMILAR SOLUTION}

Eq. (17) can be solved using the method dimensional analysis [35, 36, 53. This is performed in detail in Appendix A. The result is a self similar solution whose independent dimensionless coordinate is:

$$
\xi=\frac{r}{\left(Q^{n} A t\right)^{\frac{1}{p}}},
$$

and the solution is given in terms of a self-similar profile:

$$
w(r, t)=\left(\frac{Q^{2-k-m}}{(A t)^{d-m}}\right)^{\frac{1}{p}} f(\xi)
$$

where the self similar exponent is:

$$
p=2-k-m+(d-m) n .
$$

By substituting the self-similar solution $(22)-(23)$ in the diffusion Eq. (17), and noting that:

$$
\frac{\partial \xi}{\partial r}=\frac{\xi}{r}=\frac{1}{\left(Q^{n} A t\right)^{\frac{1}{p}}},
$$

$$
\frac{\partial \xi}{\partial t}=-\frac{1}{p} \frac{\xi}{t}
$$




$$
\frac{\partial w}{\partial r}=\left(\frac{Q^{2-k-m}}{(A t)^{d-m}}\right)^{\frac{1}{p}} f^{\prime}(\xi) \frac{\xi}{r}
$$

all dimensional quantities are factored out, and a dimensionless second order ordinary differential equation (ODE) for the self-similar solution is obtained:

$$
\frac{d}{d \xi}\left(\xi^{k+d-1} f^{n}(\xi) \frac{d f}{d \xi}+\frac{\xi^{d-m}}{p} f(\xi)\right)=0
$$

By substituting the self-similar variables $(22)-(23)$ in Eq. (19), a dimensionless conservation equation in terms of the dimensionless variables is obtained:

$$
\mathcal{A}_{d} \int_{0}^{\infty} f(\xi) \xi^{d-m-1} d \xi=1 .
$$

Similarly, the boundary conditions for the energy density at infinity (Eq. (20) ) and the flux at the origin (Eq. (21)), are written in terms of the self-similar solution, respectively, as:

$$
\begin{gathered}
{\left[\xi^{-m} f(\xi)\right]_{\xi \rightarrow \infty}=0} \\
{\left[\xi^{k+d-1} f^{n}(\xi) \frac{d f}{d \xi}\right]_{\xi \rightarrow 0}=0 .}
\end{gathered}
$$

A direct integration of Eq. 28 gives the first order ODE:

$$
f^{n-1}(\xi) \frac{d f}{d \xi}=-\frac{\xi^{1-k-m}}{p},
$$

where, by virtue of the boundary condition (31), the constant of the integration is set to zero. Using Eq. (27) with the derivative $f^{\prime}(\xi)$ taken from Eq. (32), results in the following expression for the energy flux (18):

$$
F(r, t)=\frac{A r^{k-1} w^{n+1}(r, t) \xi^{2-k-m}}{p f^{n}(\xi)} .
$$

The solution of Eq. (32) takes two different forms - for $n>0$, which corresponds to nonlinear heat conduction, and for $n=0$, which corresponds to linear conduction. These will be analyzed in detail in the next two sections.

\section{A. Heat front propagation}

We note that from Eq. 22, it is evident that heat propagates according to $r_{h}(t) \propto t^{\frac{1}{p}}$. Therefore, in order for heat to propagate outwards, we must always have:

$$
p>0
$$

This gives an upper limit for the value of $\omega$ :

$$
\omega_{\max }=\frac{2+d n}{1+\lambda+(1-\mu)(1+n)} .
$$

In addition, the propagation speed is decreasing with time for $\omega<\omega_{\text {acc }}$, constant for $\omega=\omega_{\text {acc }}$ and increasing with time for $\omega>\omega_{\text {acc }}$, where:

$$
\omega_{\mathrm{acc}}=\frac{1+d n}{2+d n} \omega_{\max } .
$$

It is interesting to note that since $0<\omega_{\text {acc }}<\omega_{\max }$, the well known solution to the nonlinear diffusion equation with constant density $(\omega=0)$, always has a decelerating speed of propagation, as $r_{h}(t) \propto t^{\frac{1}{2}}$ for linear heat conduction and $r_{h}(t) \propto t^{\frac{1}{2+d n}}$ for nonlinear heat conduction [34-36]. Therefore, we see that for a large enough value of $\omega$, due to a spatial increase in the diffusion coefficient (Eq. (7)), the heat propagation speed can be constant or even accelerate. This phenomena will be demonstrated below, in Fig. 13 .

\section{B. Relation to the strong explosion shock}

The shock trajectory for a point explosion [12, 4751, 54, 55, when heat conduction is negligible, behaves as $r_{\text {shock }}(t) \propto t^{\frac{2}{2+d-\omega}}$. This $\omega$ dependent shock trajectory is often compared [12], for $\omega=0$, to the heat wave trajectory, $r_{h}(t) \propto t^{\frac{1}{2+d n}}$. It is concluded that at short times, the heat wave travels faster than the shock, so that the hydrodynamic motion is negligible and the heat wave is considered to be "supersonic", while at longer times, the heat front slows down (becomes "subsonic"), and is overtaken by the shock. However, it is now evident that this common picture can change, depending on the value of $\omega$. If $\frac{1}{p}<\frac{2}{2+d-\omega}$, we have the common case of a supersonic heat wave which is overtaken by a shock, while for $\frac{1}{p}>\frac{2}{2+d-\omega}$, we have a pure hydrodynamic shock at short times, which is overtaken by a heat wave at longer times. The resulting critical spatial exponent for this transition is:

$$
\bar{\omega}=\frac{(2+d(2 n-1)) \omega_{\max }}{2(2+d n)-\omega_{\max }}
$$

so that for $\omega<\bar{\omega}$ we have the common heat wave followed by a shock behavior, while for $\omega>\bar{\omega}$, the order is revered. For $\omega=\bar{\omega}$, both waves propagate at the same speed, $r_{\text {shock }}(t) / r_{h}(t)=$ const, and a full rad-hydro self-similar solution may be obtained (as was already noted in Ref. [12] for $\omega=0)$.

\section{Relation to Marshak waves}

The temperature profile is given by:

$$
T(r, t)=\left(\frac{w(r, t)}{f \rho_{0}^{1-\mu}}\right)^{\frac{1}{\beta}}
$$




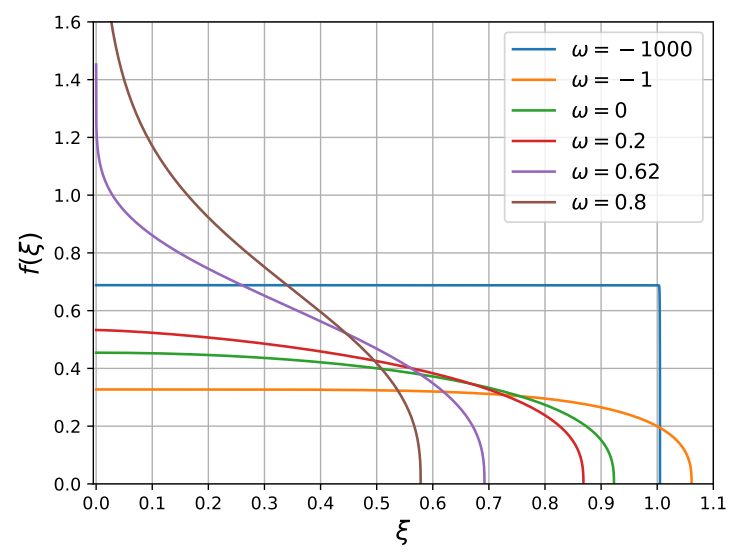

Figure 1. Various forms of the self-similar solution $f(\xi)$ for different values of $\omega$ (listed in the legend), for the following parameters: $d=3$ (spherical symmetry), $n=2.75$ (nonlinear conduction), $\lambda=1$ and $\mu=0$.

As a result, from the self-similar form in Eq. (23), the temperature at the origin is given by:

$$
T(0, t) \equiv T_{0} t^{\tau}
$$

where:

$$
\begin{gathered}
\tau=-\left(\frac{d-m}{\beta p}\right), \\
T_{0}=\left(\frac{f(\xi \rightarrow 0)}{f \rho_{0}^{1-\mu}}\left(\frac{Q^{2-k-m}}{A^{d-m}}\right)^{\frac{1}{p}}\right)^{\frac{1}{\beta}} .
\end{gathered}
$$

As a result, for planar symmetry $(d=1)$, the solution presented here is essentially a special analytic solution of the corresponding Marshak supersonic heat wave [25, 28, 31, 52, 56, 57, with an imposed boundary temperature of the form $T_{b}(t)=T_{0} t^{\tau}$, and a density profile of the form $\rho(r)=\rho_{0} r^{-\omega}$.

\section{NONLINEAR CONDUCTION}

Assuming $n>0$, and employing the boundary condition at infinity (see Eq. (30), the ODE in Eq. (32) has a simple analytic solution of the form:

$$
f(\xi)= \begin{cases}\left(\frac{n\left(\xi_{0}^{2-k-m}-\xi^{2-k-m}\right)}{p(2-k-m)}\right)^{\frac{1}{n}}, & \xi<\xi_{0} \\ 0, & \xi>\xi_{0}\end{cases}
$$

where $\xi_{0}$ is a constant, which represents the self similar coordinate of the heat wave position. Hence, the heat wave position as a function of time is given by:

$$
r_{h}(t)=\xi_{0}\left(Q^{n} A t\right)^{\frac{1}{p}}
$$
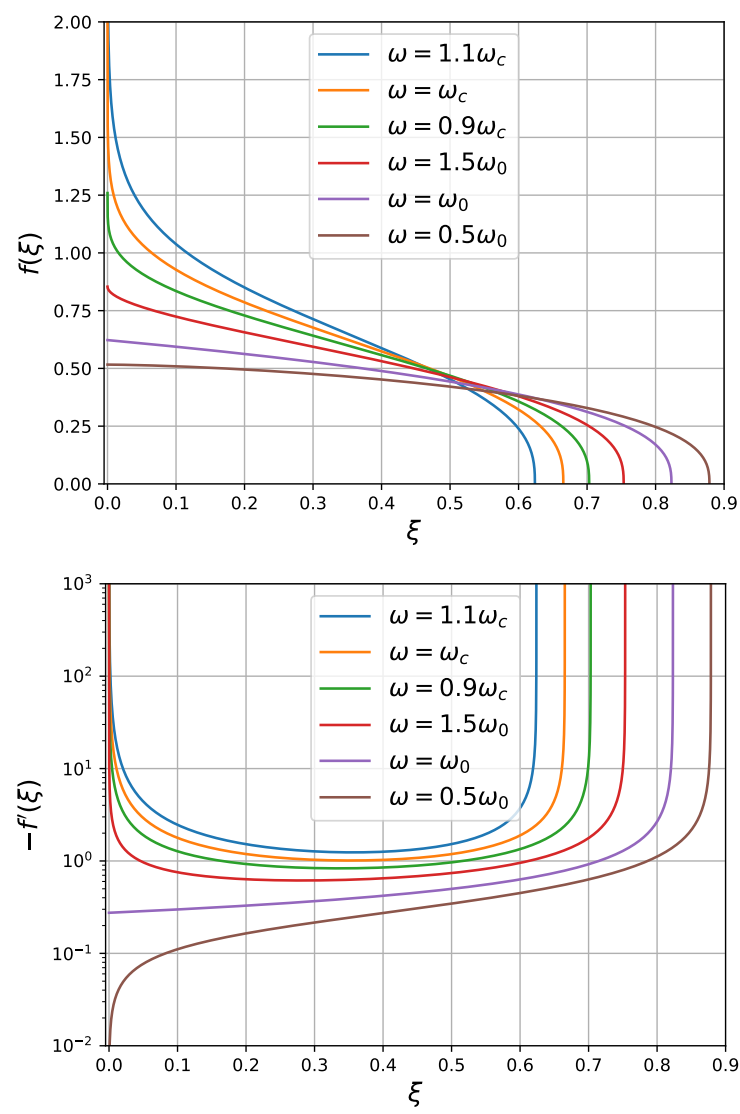

Figure 2. The self-similar solution $f(\xi)$ (upper figure) and its derivative $-f^{\prime}(\xi)$ (lower figure) for different values of $\omega$ in the various $\omega>0$ ranges (see table I), for the same parameters as in Fig. 1 (for which $\omega_{\max }=1.7826, \omega_{c}=\frac{2}{3}$ and $\left.\omega_{0}=\frac{1}{3}\right)$. The solutions are plotted for the following values: $\omega=1.1 \omega_{c}=\frac{11}{15}$ (blue lines), $\omega=\omega_{c}=\frac{2}{3}$ (orange lines), $\omega=0.9 \omega_{c}=0.6$ (green lines), $\omega=1.5 \omega_{0}=0.5$ (red lines), $\omega=\omega_{0}=\frac{1}{3}$ (purple lines) and $\omega=0.5 \omega_{c}=\frac{1}{6}$ (brown lines).

The value of $\xi_{0}$ can be found by substituting the solution (42) in the energy conservation constraint (29). The resulting value of $\xi_{0}$, which is derived in detail in Appendix $B$, is:

$$
\xi_{0}=\left(\frac{p|2-k-m|^{n+1}}{n \mathcal{A}_{d}^{n} \mathcal{B}^{n}\left(l, \frac{1}{n}+1\right)}\right)^{\frac{1}{p}}
$$

where the first beta function argument is:

$$
l= \begin{cases}\frac{d-m}{2-k-m}, & 2-k-m>0 \\ -\frac{1}{n}-\frac{d-m}{2-k-m}, & 2-k-m<0\end{cases}
$$

The solution in Eq. 42 is valid only under the constraint $d-m>0$ and (34) (which is equivalent to $\omega<\omega_{\max }$ ).

It is evident that for the constant density case $(\omega=$ $k=m=0)$, the solution $(42)$ is reduced to the well known nonlinear heat wave [34,36, 57, which has a sharp 


\begin{tabular}{|c|c|c|c|c|c|c|c|}
\hline & $\omega \rightarrow-\infty$ & $\omega<\omega_{0}$ & $\omega=\omega_{0}$ & $\omega_{0}<\omega<\omega_{c}$ & $\omega=\omega_{c}$ & $\omega_{c}<\omega<\omega_{\max }$ & $\omega \rightarrow \omega_{\max }^{-}$ \\
\hline \hline$\xi_{0}$ & 1 & finite & finite & finite & finite & finite & 0 \\
\hline$f(0)$ & $\infty$ & finite & finite & finite & $\infty$ & $\infty$ & $\infty$ \\
\hline$f^{\prime}(0)$ & 0 & 0 & finite & $-\infty$ & $-\infty$ & $-\infty$ & $-\infty$ \\
\hline
\end{tabular}

Table I. Behavior of the self-similar heat front coordinate $\xi_{0}$ and the self-similar solution $f(0)$ and its derivative $f^{\prime}(0)$ at the origin, for various ranges of the spatial density power $\omega$, for nonlinear heat conduction $(n>0)$.

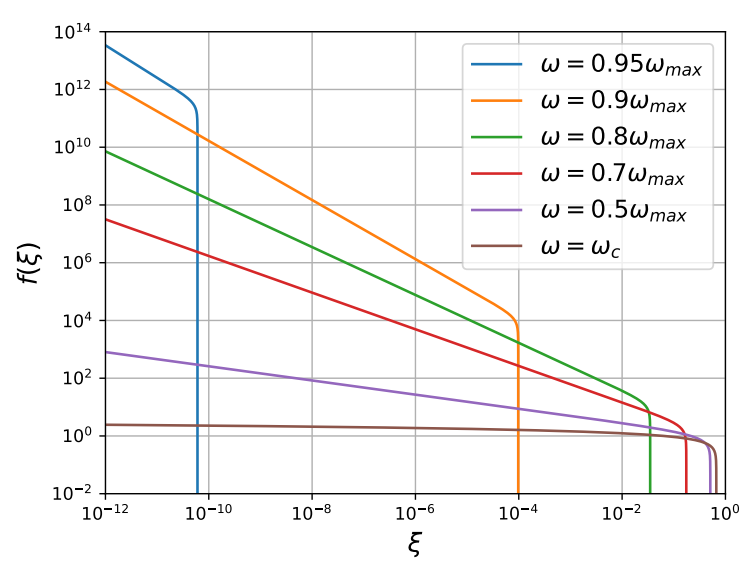

Figure 3. The self-similar solution $f(\xi)$ for different values of $\omega$ near $\omega_{\max }$, for the same parameters of Fig. 1 $\left(\omega_{\max }=1.7826\right)$. The solutions are plotted for the following values: $\omega=0.95 \omega_{\max }=1.693$ (blue line), $\quad \omega=0.9 \omega_{\max }=1.604 \quad$ (orange line), $\omega=0.8 \omega_{\max }=1.426 \quad$ (green line), $\omega=0.7 \omega_{\max }=1.248$ (red line), $\omega=0.5 \omega_{\max }=0.891$ (purple line) and $\omega=\omega_{c}=\frac{2}{3}$ (brown line).

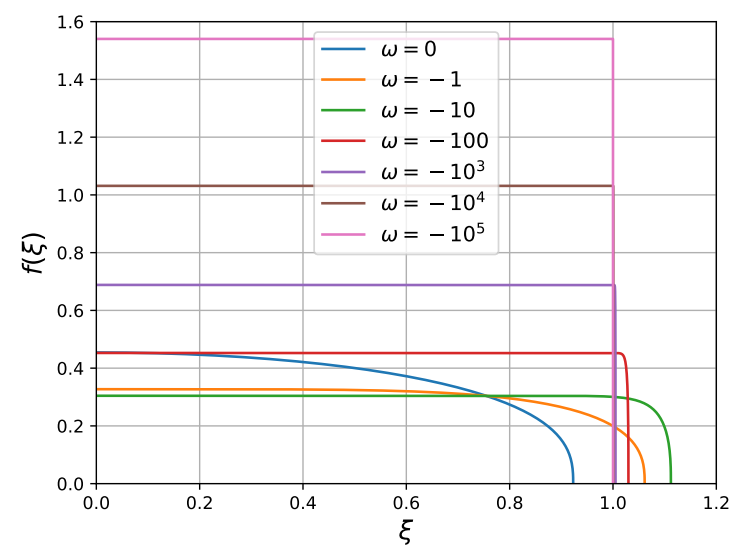

Figure 4. The self-similar solution $f(\xi)$ for decreasing values of $\omega$ (listed in the legend), for the same parameters of Fig. 1 (nonlinear conduction).

front at $\xi \rightarrow \xi_{0}$, and takes the form $f(\xi) \propto\left(\xi_{0}^{2}-\xi^{2}\right)^{\frac{1}{n}}$, for which $f^{\prime}(0)=0$, and the solution approaches a finite constant value near the origin. However, for a nonhomogeneous media ( $\omega \neq 0$, which results in $k, m \neq 0)$, which is considered in this work, this form is general- ized to $f(\xi) \propto\left(\xi_{0}^{2-k-m}-\xi^{2-k-m}\right)^{\frac{1}{n}}$, which may differ qualitatively from the traditional $\omega=0$ solution. In fact, as demonstrated in Fig. 11, several different qualitative forms take place, according to the value of $\omega$. It is seen that: (i) for large enough values of $\omega$, the solution diverges at the origin, (ii) for smaller values of $\omega$ the solution is finite at the origin but its derivative diverges, (iii) for smaller values of $\omega$ the solution approaches a constant near the origin (a familiar property of the solution for $\omega=0$ ), and finally (iv) for large negative values of $\omega$ the solution becomes steeper and approaches a step function, where the step value diverges as $\omega \rightarrow-\infty$. These different forms shown in Fig. 11, will be analyzed in detail below, and are summarized in table $\mathbb{I}$.

\section{A. Analysis of various solution forms for different values of $\omega$}

First we note that since $f^{\prime}(\xi \rightarrow 0) \propto \xi^{1-k-m}$. For $1-$ $k-m<0$ one has $f^{\prime}(0) \rightarrow-\infty$, so that the solution slope diverges near the origin. This condition is equivalent to $\omega>\omega_{0}$, where:

$$
\omega_{0}=\frac{1}{2+\lambda-\mu} .
$$

For the special value $\omega=\omega_{0}$, the value of $f^{\prime}(0)$ is finite and can be obtained from Eq. (32), so that we have:

$$
f^{\prime}(0)= \begin{cases}-\infty, & \omega>\omega_{0} \\ -\frac{1}{p f^{n-1}(0)}, & \omega=\omega_{0} \\ 0, & \omega<\omega_{0}\end{cases}
$$

Moreover, the solution 42 is valid even for $2-k-m<0$ (provided that Eq. (34) holds), for which the self similar profile itself diverges at the origin. The latter condition is equivalent to $\omega>\omega_{c}$, where:

$$
\omega_{c}=2 \omega_{0}
$$

We also note that for the special value $\omega=\omega_{c}$, the solution in equations 42,44 and 45 cannot be used directly ( since $2-k-m=0$ ). However, the ODE (32) can be solved independently for this case, as done in detail in Appendix C. The resulting solution takes the marginal form: 

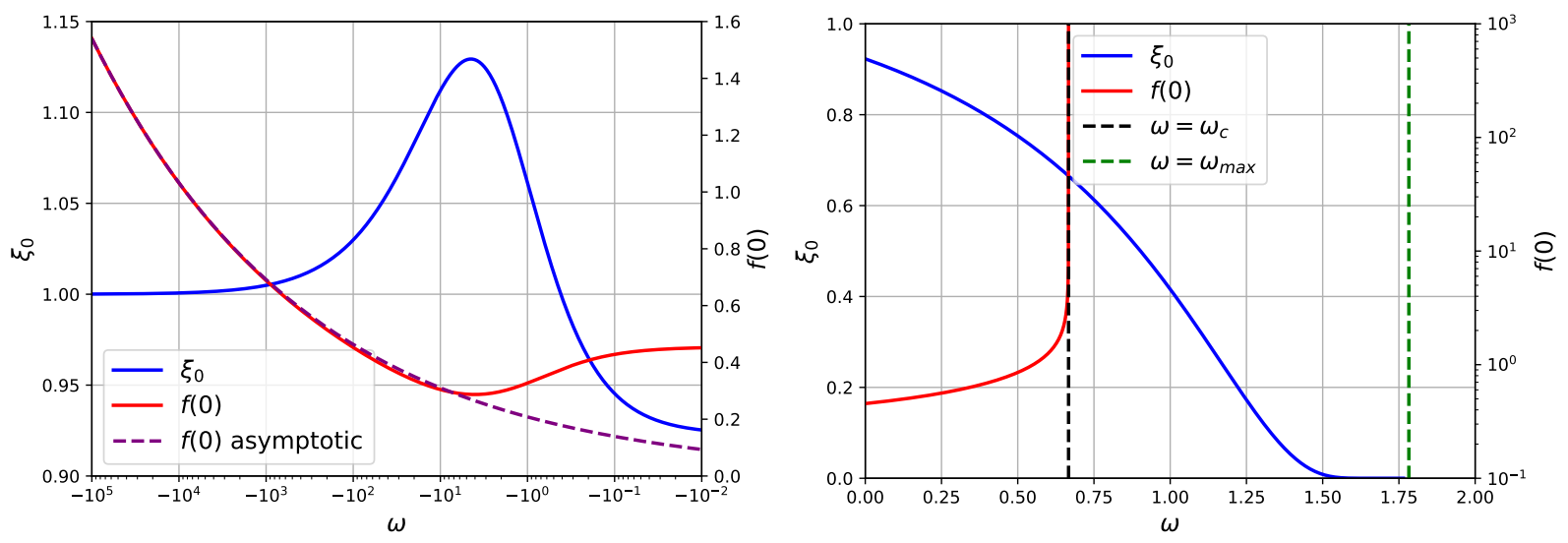

Figure 5. The values of the self-similar heat front coordinate $\xi_{0}$ (in blue, on the left y axes) and $f(0)$ (in red, on the right y axes), as a function of $\omega$. The problem's parameters are the same as in Fig. 1 (nonlinear conduction). Negative $\omega$ values, in a wide range, are plotted on the left figure, while positive values are plotted on the right figure. The asymptotic approximation (Eq. (53) ) of $f(0)$ for $\omega \rightarrow-\infty$ is given in the purple dashed line. The special limiting values $\omega_{c}$ and $\omega_{\max }$ (see table I), are given by the black and green vertical lines, respectively.

$$
f(\xi)= \begin{cases}{\left[\frac{1}{d-m} \ln \left(\frac{\xi_{0}}{\xi}\right)\right]^{\frac{1}{n}},} & \xi<\xi_{0} \\ 0, & \xi>\xi_{0}\end{cases}
$$

where:

$$
\xi_{0}=\left[\frac{(d-m)^{1+\frac{2}{n}}}{\mathcal{A}_{d} \Gamma\left(1+\frac{1}{n}\right)}\right]^{\frac{1}{d-m}} .
$$

The solution $f(\xi)$ and its derivative $f^{\prime}(\xi)$ are shown in Fig. 2 for various values of $\omega$ (see table $\bar{I}$ : $\omega_{c}<\omega<\omega_{\max }$ (diverging solution and derivative at the origin), $\omega=\omega_{c}$ (marginal form (49)), $\omega_{0}<\omega<\omega_{c}$ (finite solution and diverging derivative at the origin), $\omega=\omega_{0}$ (finite solution and derivative at the origin) and $\omega<\omega_{0}$ (derivative is zero at the origin).

Fig. 3 shows various solutions for $\omega \geq \omega_{c}$ close to $\omega_{\max }$. The divergence of these solutions near the origin is evident, as well as the fact that:

$$
\lim _{\omega \rightarrow \omega_{\max }^{-}} \xi_{0}=0
$$

which is straightforward to show directly from Eq. (44), or from the fact that energy conservation 290 must hold. This is also shown in Fig. 5, where the values of $\xi_{0}$ and $f(0)$ are plotted as a function of $\omega$.

Finally, we consider the interesting limiting solution for $\omega \rightarrow-\infty$. First we note that it is straightforward to calculate that limit of Eq. 44, which is:

$$
\lim _{\omega \rightarrow-\infty} \xi_{0}=1,
$$

and to show that the resulting limiting solution 42 has a step function form:

$$
\lim _{\omega \rightarrow-\infty} f(\xi)= \begin{cases}B|\omega|^{b}, & \xi<1 \\ 0, & \text { else }\end{cases}
$$

where:

$$
b=\frac{\lambda+\mu}{2+d n} \omega_{\max },
$$

and:

$$
B=\frac{\left(\omega_{0}^{1-q(n+1)}\left(\frac{n \omega_{\max }}{2+d n}\right)^{1-q}\right)^{\frac{1}{n}}}{\left(\mathcal{A}_{d} \mathcal{B}\left(\bar{l}, \frac{1}{n}+1\right)\right)^{q}},
$$

where:

$$
q=\frac{\omega_{\max }}{\omega_{0}(2+d n)},
$$

and the $\omega \rightarrow-\infty$ limit of Eq. 45 is:

$$
\bar{l}=(1-\mu) \omega_{0}
$$

We note that since $b>0$, the step function diverges as $\omega \rightarrow-\infty$, which is of course necessary in order for the energy conservation constraint $(29)$ to hold. The steepening of the solution for increasing values of $\omega$ as well as the divergence of the step value is demonstrated in Fig. 4 The limit (52) and the asymptotic form (53) are demonstrated in Fig. 5

Figs 1.5 are calculated for the following case study: $d=3$ (spherical symmetry), $n=2.75, \lambda=1$ and $\mu=0$, for which $\omega_{\max }=1.7826, \omega_{c}=\frac{2}{3}$ and $\omega_{0}=\frac{1}{3}$. 


\section{LINEAR CONDUCTION}

Assuming $n=0$, which corresponds to the linear conduction case, the solution of Eq. 32, has a superGaussian form:

$$
f(\xi)=f_{0} \exp \left(-\frac{\xi^{2-k-m}}{(2-k-m)^{2}}\right) .
$$

As in the nonlinear case, the constant $f_{0}$ is obtained from the energy conservation constraint 29):

$$
f_{0}=\frac{1}{\mathcal{A}_{d} \Gamma\left(\frac{d-m}{2-k-m}\right)(2-k-m)^{\frac{2(d-m)+k+m-2}{2-k-m}}}
$$

where we have employed the well known identity:

$$
\int_{0}^{\infty} x^{b} \exp \left(-c x^{a}\right) d x=\frac{\Gamma\left(\frac{b+1}{a}\right)}{a c^{\frac{b+1}{a}}} .
$$

As in the nonlinear conduction case, the solution in equations (58)-59 is valid only under the constraint (34), which is now equivalent to $\omega<\omega_{\max }=\omega_{c}$. As a result, this solution also obeys the boundary condition 30 .

It is evident that for the constant density case $\omega=$ $k=m=0)$, the solution $(58)-(59)$ is reduced to the well known Gaussian solution to the linear diffusion equation:

$$
f(\xi)=\frac{1}{2^{d} \pi^{\frac{d}{2}}} e^{-\frac{\xi^{2}}{4}}, \xi=\frac{r}{(A t)^{\frac{1}{2}}},
$$

for which $f^{\prime}(0)=0$, and the solution approaches a finite constant value near the origin. However, similarly to the non-linear conduction case that was considered in the previous section, for a non-homogeneous media $(\omega \neq 0$, which results in $k, m \neq 0$ ), this form is generalized to $f(\xi) \propto e^{-\xi^{2-k-m}}$, which may differ qualitatively from the traditional $\omega=0$ solution. This is demonstrated in Fig. 6, where several different qualitative forms are shown for different values of $\omega$. These different forms will be analyzed in detail below, and are summarized in table II

\section{A. Analysis of various solution forms for different values of $\omega$}

As in the nonlinear conduction case, Eq. (58) gives $f^{\prime}(\xi \rightarrow 0) \propto \xi^{1-k-m}$, so that behavior of the derivative near the origin is the same as in the nonlinear case, and specifically, Eq. (47) is applicable for $n=0$ as well.

Similarly, Eq. (58) gives $f(\xi \rightarrow 0) \propto \xi^{2-k-m}$, but since $\omega<\omega_{\max }=\omega_{c}$ for $n=0$ (equivalent to $2-k-$ $m>0$ ), we see that the solution is always finite at the origin for linear conduction. The solution $f(\xi)$ and its derivative $f^{\prime}(\xi)$ are shown in Fig. 7 for various values

\begin{tabular}{|c|c|c|c|c|c|}
\hline & $\omega \rightarrow-\infty$ & $\omega<\omega_{0}$ & $\omega=\omega_{0}$ & $\omega_{0}<\omega<\omega_{\max }$ & $\omega \rightarrow \omega_{\max }^{-}$ \\
\hline \hline$f(0)$ & $\infty$ & finite & finite & finite & $\infty$ \\
\hline$f^{\prime}(0)$ & 0 & 0 & finite & $-\infty$ & $-\infty$ \\
\hline
\end{tabular}

Table II. Behavior of the the self-similar solution $f(0)$ and its derivative $f^{\prime}(0)$ at the origin, for various ranges of the spatial density power $\omega$, for linear conduction $(n=0)$.

of $\omega$ (see table II): $\omega_{0}<\omega<\omega_{c}$ (finite solution and diverging derivative at the origin), $\omega=\omega_{0}$ (finite solution and derivative at the origin) and $\omega<\omega_{0}$ (derivative is zero at the origin).

Fig. 8 shows various solutions for $\omega \geq \omega_{0}$ close to $\omega_{\max }$. It is seen that these solutions are more concentrated near the origin for $\omega$ closer to $\omega_{\max }$ and that:

$$
\lim _{\omega \rightarrow \omega_{\max }^{-}} f(0)=\infty
$$

as can be shown directly from Eq. (59), or from the fact that energy conservation (29) must hold. This is also shown in Fig. 10, where the values of $\xi_{0}$ and $f(0)$ are plotted as a function of $\omega$.

Finally, we consider the limiting solution for $\omega \rightarrow-\infty$. It is straightforward to show that the resulting limiting solution (58), has the same step function form as in the nonlinear case, Eq. (53), with $b=\omega_{0}(\lambda+\mu)$ (which is the same as Eq. (54) for $n=0$ ), and:

$$
B=\frac{1}{\mathcal{A}_{d} \omega_{0}^{b} \Gamma\left((1-\mu) \omega_{0}\right)} .
$$

As noted for the nonlinear case, the step function diverges as $\omega \rightarrow-\infty$, which is of course necessary in order for the energy conservation constraint (29) to hold. The steepening of the solution for increasing values of $\omega$ as well as the divergence of the step value is demonstrated in Fig. 9. The asymptotic form (53) (using Eq. (63)) is shown in Fig. 10 and agrees well with the solution for $\omega \rightarrow-\infty$.

Figs 6-10 are calculated for the following case study: $d=3$ (spherical symmetry), $n=0, \lambda=1$ and $\mu=0$, for which $\omega_{\max }=\omega_{c}=\frac{2}{3}$, and $\omega_{0}=\frac{1}{3}$.

\section{COMPARISON WITH SIMULATIONS}

In order to test the new analytic solutions presented in this work and to demonstrate their utility for verification and validation of computer simulations, we have performed detailed numerical simulations of the radiation diffusion equation (1) for both nonlinear and linear conduction and for various values of $\omega$. The simulations use a standard one dimensional fully implicit nonlinear diffusion scheme, and temperature time step control with a tolerance of $1 \%$. All simulations are performed for spherical symmetry $(d=3)$, using 1000 computational cells, with power law opacity (Eq. (4) with $g=1$ ), and EOS 


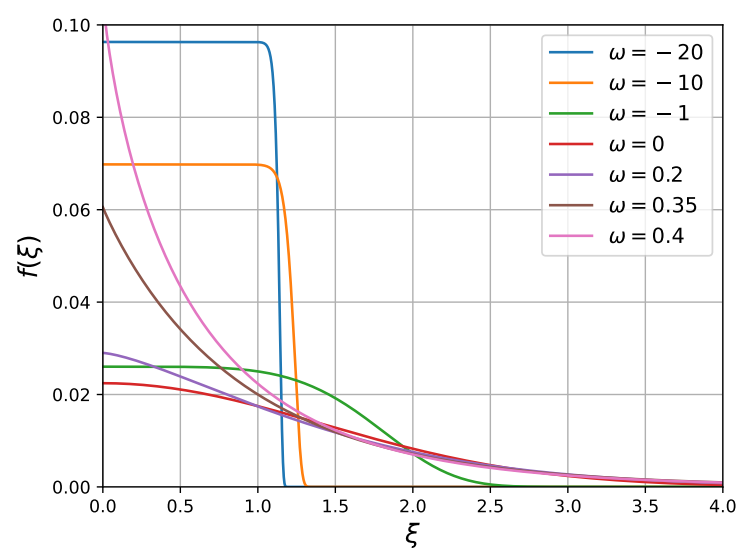

Figure 6. Various forms of the self-similar solution $f(\xi)$ for different values of $\omega$ (listed in the legend), for the following parameters: $d=3$ (spherical symmetry), $n=0$ (linear conduction), $\lambda=1$ and $\mu=0$.
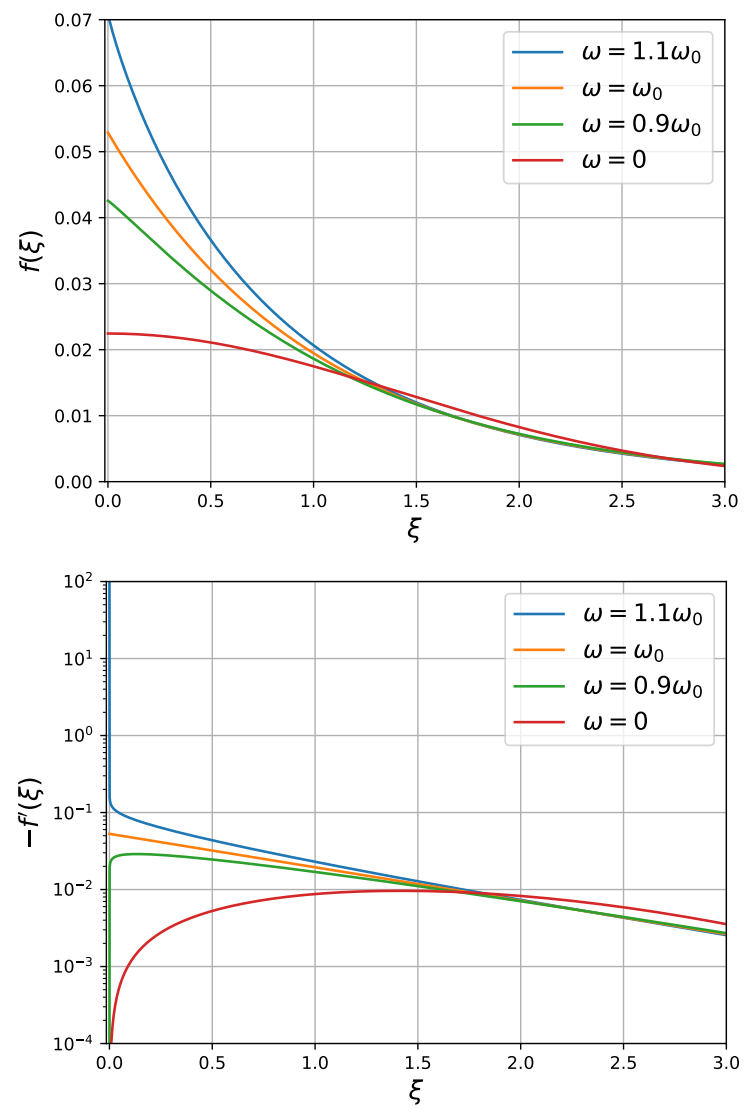

Figure 7. The self-similar solution $f(\xi)$ (upper figure) and its derivative $-f^{\prime}(\xi)$ (lower figure) for different values of $\omega$ (see table II), for the same parameters of Fig. 6] (for which $\omega_{c}=\omega_{\max }=\frac{2}{3}$ and $\left.\omega_{0}=\frac{1}{3}\right)$. The solutions are plotted for the following values: $\omega=1.1 \omega_{0}=\frac{11}{30}$ (blue lines), $\omega=\omega_{0}=\frac{1}{3}$ (orange lines), $\omega=0.9 \omega_{0}=0.3$ (green lines), $\omega=0$ (red lines).

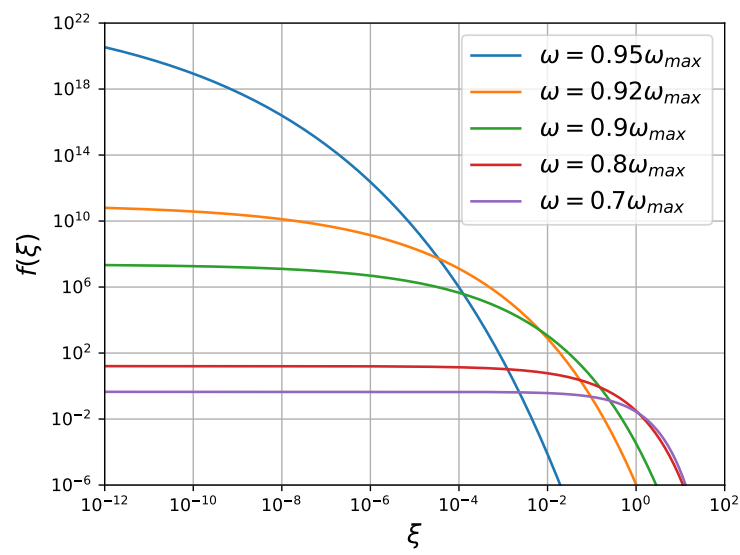

Figure 8. The self-similar solution $f(\xi)$ for different values of $\omega$ near $\omega_{\max }$, for the same parameters of Fig. 6 $\left(\omega_{\max }=\frac{2}{3}\right)$. The solutions are plotted for the following values: $\omega=0.95 \omega_{\max }=0.633$ (blue line), $\omega=0.92 \omega_{\max }=0.613 \quad$ (orange line),$\quad \omega=0.9 \omega_{\max }=0.6$ (green line), $\quad \omega=0.8 \omega_{\max }=0.533 \quad$ (red line) and $\omega=0.7 \omega_{\max }=0.466$ (purple line).

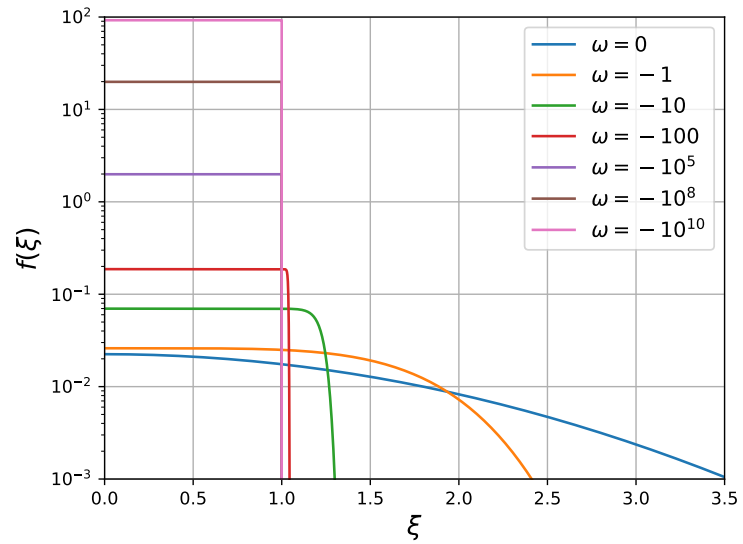

Figure 9. The self-similar solution $f(\xi)$ for increasing values of $\omega$, for the same parameters of Fig. 6 (linear conduction).

(Eq. (5) with $f=1$ ), and are initialized with a spatial power law density (Eq. (6) with $\rho_{0}=1$ ), and with a total energy of $Q=1$ deposited in the first cell (and zero energy elsewhere), in order to represent the instantaneous energy point source. All quantities are given in c.g.s. units.

In Fig. 11, the numerical simulations are compared with the analytic solutions for nonlinear conduction given in Eq. (42) for the parameters $\alpha=2, \beta=1.6, \lambda=1$ and $\mu=0$, for which $n=2.75, \omega_{\max }=1.7826, \omega_{\text {acc }}=$ 1.6087, $\bar{\omega}=1.4762, \omega_{c}=\frac{2}{3}$ and $\omega_{0}=\frac{1}{3}$ (which are the same parameters of the nonlinear conduction case studied in Figs. 1 15). Temperature profiles are shown at different times such that the nonlinear heat wave (Eq. (43) reaches radii of $r=0.2,0.5,0.7$ and 0.9 . The values of $\omega$ were chosen in order to present the different 

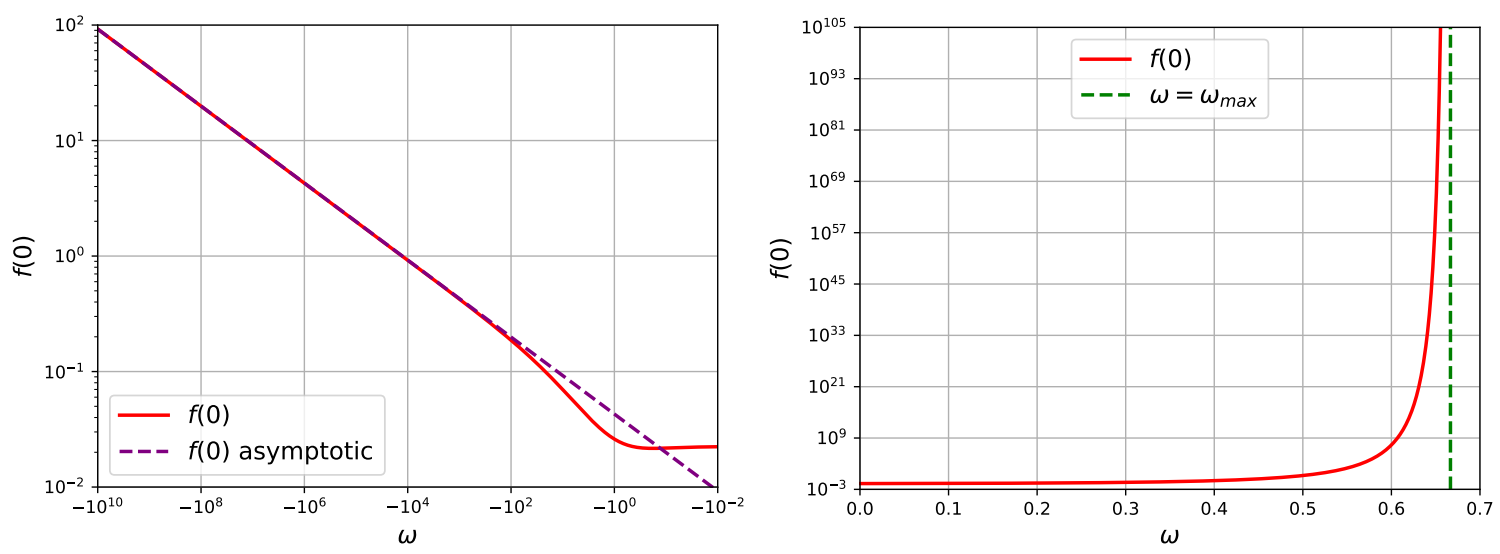

Figure 10. The values of the self-similar solution at the origin, $f(0)$ (in red), as a function of $\omega$. The problem's parameters are the same as in Fig. 6 (linear conduction). Negative $\omega$ values, in a wide range, are plotted on the left figure, while positive values are plotted on the right figure. The asymptotic approximation (equations (53), (63)) of $f(0)$ for $\omega \rightarrow-\infty$ is given in the purple dashed line. The special limiting value $\omega_{\max }$ (see table II), is shown in the vertical line.
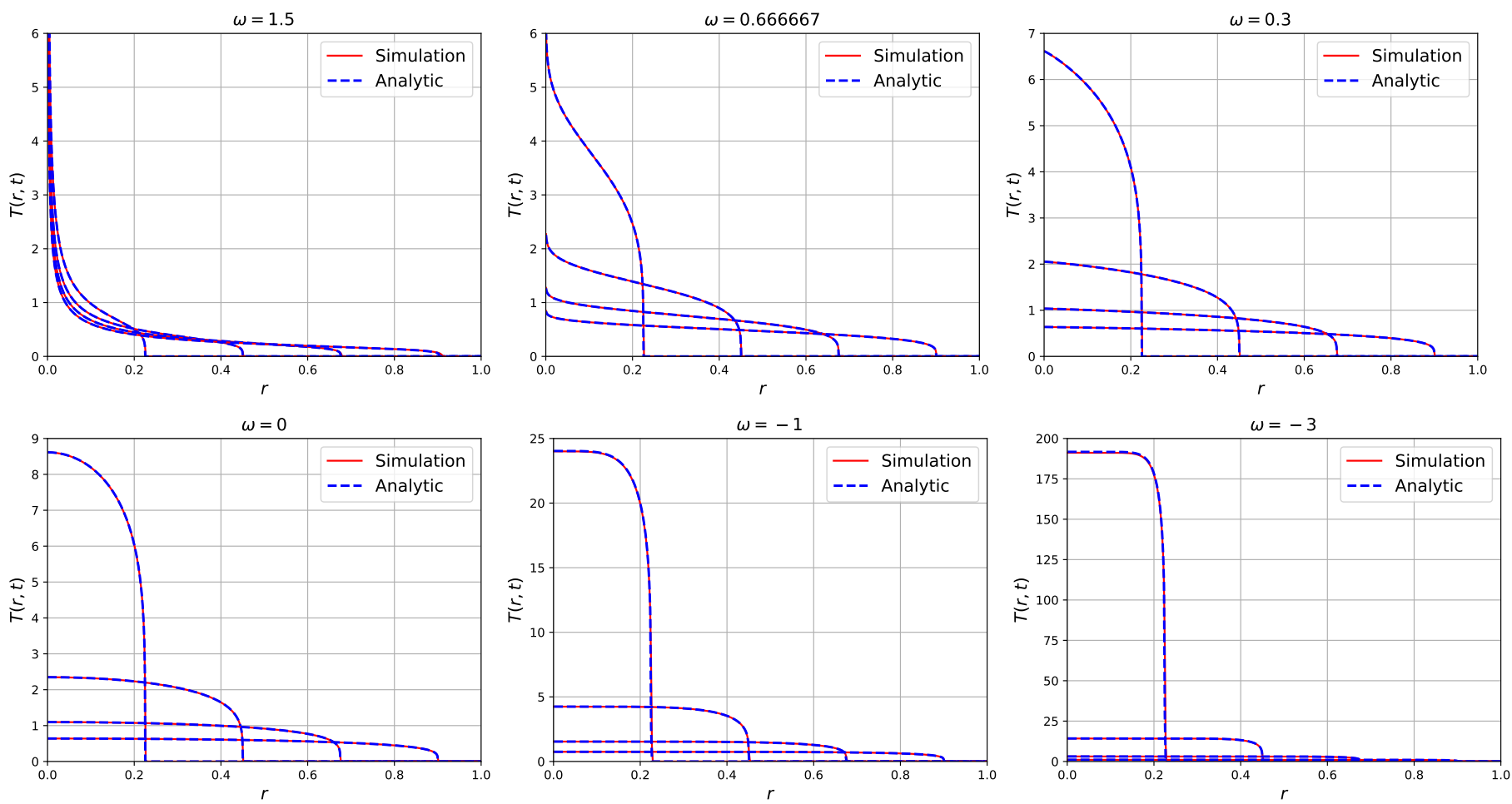

Figure 11. A comparison between numerical simulations (red lines) and analytic solutions (blue dashed lines) of the radiation diffusion equation (1) with an instantaneous point source for various values of $\omega$ (given in the title of each sub-figure). Temperature profiles (in Kelvin) are shown at different times such that the heat wave reaches radii of $r=0.225,0.45,0.675$ and 0.9. The comparisons are performed for $d=3$ (spherical symmetry), $\alpha=2, \beta=1.6, \lambda=1$ and $\mu=0$, for which $n=2.75$ (nonlinear conduction), $\omega_{\max }=1.7826, \omega_{\mathrm{acc}}=1.6087, \omega_{c}=\frac{2}{3}$ and $\omega_{0}=\frac{1}{3}$. The energy deposited at the origin at $t=0$ is $Q=1$. All quantities are given in c.g.s. units.

solution forms, according to table I] A comparison for the special value $\omega=\omega_{c}$ is also shown, which validates and demonstrates the marginal self-similar solution in Eq. (49). Finally, we note that the steepening of the heat wave towards a step function is evident for increasing negative values of $\omega$.

Similarly, in Fig. 12 the numerical simulations are compared with the analytic solutions for linear conduction given in Eq. (58), for the parameters $\alpha=1, \beta=5$, $\lambda=1$ and $\mu=0$, for which $n=0, \omega_{\max }=\omega_{c}=\frac{2}{3}$, and 

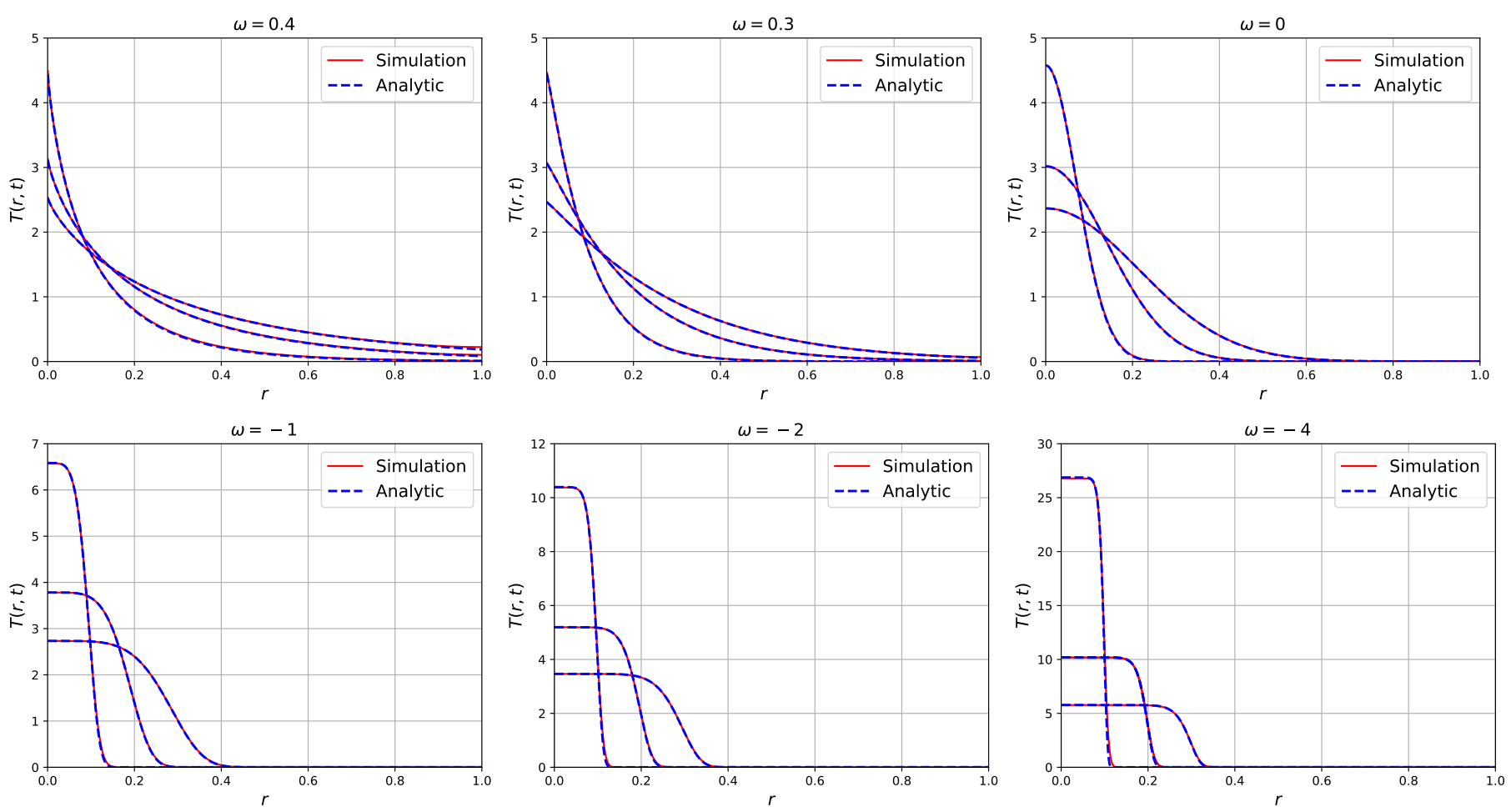

Figure 12. A comparison between numerical simulations (red lines) and analytic solutions (blue dashed lines) of the radiation diffusion equation (1) with an instantaneous point source for various values of $\omega$ (given in the title of each sub-figure). Temperature profiles are shown at different times such that the argument of the exponent in Eq. (58) equals $0.1333,0.2667$ and 0.4 . The comparisons are performed for $d=3$ (spherical symmetry), $\alpha=1$ and $\beta=5, \lambda=1$ and $\mu=0$, for which $n=0$ (linear conduction), $\omega_{\max }=\omega_{c}=\frac{2}{3}$, and $\omega_{0}=\frac{1}{3}$.

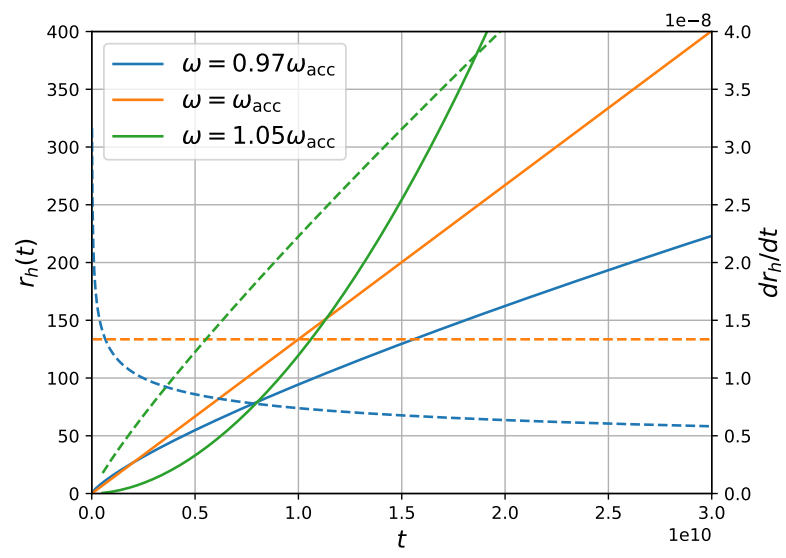

Figure 13. The heat wave position in Eq. 43) (left y axis, solid lines) and velocity (right y axis, dashed lines) as a function of time, for the same parameters of Fig. 11 (nonlinear conduction), and for $\omega=0.97 \omega_{\text {acc }}=1.56$ (decelerating heat front, in blue), $\omega=\omega_{\text {acc }}=1.609$ (constant speed heat front, in orange) and $\omega=1.05 \omega_{\mathrm{acc}}=1.689$ (accelerating heat front, in orange).

$\omega_{0}=\frac{1}{3}$ (which are the same parameters of the linear conduction case studied in Figs. 6 100. Temperature profiles are shown at different times such that the argument of the exponent in Eq. (58) equals 0.1333, 0.2667 and 0.4, which represent different positions the non-sharp linear conduction heat front. The values of $\omega$ are chosen in order to present the different solution forms, according to table II] We note that the steepening of the superGaussian heat wave towards a step function is evident for increasing negative values of $\omega$.

A comparison of the nonlinear heat front position and velocity for $\omega<\omega_{\text {acc }}, \omega=\omega_{\text {acc }}$ and $\omega>\omega_{\text {acc }}$ is shown in Fig. 13 As was argued at the end of Sec. III, it is evident that the resulting heat front propagation decelerates, has a constant speed and accelerates, respectively.

Finally, we note that we have also compared the energy fluxes given from the simulations with the analytic solutions, presented here in Eq. (33). These comparison are not presented here for the sake of brevity, but we note that a very good agreement was reached, so that Eq. (33) can also be used for the purpose of verification and validation of computer simulations.

\section{SUMMARY}

In this work we have generalized the well known solutions to the nonlinear radiation diffusion equation with an instantaneous point source, to a non-homogeneous 
medium with a power law spatial density profile. It was shown that the solutions take various qualitatively different forms according to the value of the spatial density exponent $\omega$. The different forms were studied in detail for both linear and non linear heat conduction. The various solution forms were compared in detail to numerical simulations, and a good agreement was achieved. These new solutions can be used for verification and validation of numerical simulations of the radiation diffusion equation, as well as for any diffusion model.

\section{ACKNOWLEDGMENTS}

The author thanks Shay I. Heizler for useful suggestions and comments.

\section{Availability of data}

The data that support the findings of this study are available from the corresponding author upon reasonable request.
[1] John D Lindl, Peter Amendt, Richard L Berger, S Gail Glendinning, Siegfried H Glenzer, Steven W Haan, Robert L Kauffman, Otto L Landen, and Laurence J Suter. The physics basis for ignition using indirect-drive targets on the national ignition facility. Physics of plasmas, 11(2):339-491, 2004.

[2] CA Back, JD Bauer, JH Hammer, BF Lasinski, RE Turner, PW Rambo, OL Landen, LJ Suter, MD Rosen, and WW Hsing. Diffusive, supersonic x-ray transport in radiatively heated foam cylinders. Physics of plasmas, 7(5):2126-2134, 2000.

[3] Harry F Robey, JO Kane, BA Remington, RP Drake, OA Hurricane, H Louis, RJ Wallace, J Knauer, P Keiter, D Arnett, et al. An experimental testbed for the study of hydrodynamic issues in supernovae. Physics of Plasmas, 8(5):2446-2453, 2001.

[4] James E Bailey, Taisuke Nagayama, Guillaume Pascal Loisel, Gregory Alan Rochau, C Blancard, James Colgan, Ph Cosse, G Faussurier, CJ Fontes, F Gilleron, et al. A higher-than-predicted measurement of iron opacity at solar interior temperatures. Nature, 517(7532):56-59, 2015.

[5] E Falize, Claire Michaut, and Serge Bouquet. Similarity properties and scaling laws of radiation hydrodynamic flows in laboratory astrophysics. The Astrophysical Journal, 730(2):96, 2011.

[6] OA Hurricane, DA Callahan, DT Casey, PM Celliers, Charles Cerjan, EL Dewald, TR Dittrich, T Döppner, DE Hinkel, LF Berzak Hopkins, et al. Fuel gain exceeding unity in an inertially confined fusion implosion. Nature, 506(7488):343-348, 2014.

[7] Avner P Cohen, Guy Malamud, and Shay I Heizler. Key to understanding supersonic radiative marshak waves using simple models and advanced simulations. Physical Review Research, 2(2):023007, 2020.

[8] Shay I Heizler, Tomer Shussman, and Moshe Fraenkel. Radiation drive temperature measurements in aluminum via radiation-driven shock waves: Modeling using selfsimilar solutions. Physics of Plasmas, 28(3):032105, 2021.

[9] R Sigel, R Pakula, S Sakabe, and GD Tsakiris. X-ray generation in a cavity heated by 1.3 -or $0.44-\mu \mathrm{m}$ laser light. iii. comparison of the experimental results with theoret- ical predictions for x-ray confinement. Physical Review A, 38(11):5779, 1988.

[10] Paul Keiter, Mark Gunderson, John Foster, Paula Rosen, Andrew Comley, Mark Taylor, and Ted Perry. Radiation transport in inhomogeneous media. Physics of Plasmas, 15(5):056901, 2008.

[11] John Lindl. Development of the indirect-drive approach to inertial confinement fusion and the target physics basis for ignition and gain. Physics of plasmas, 2(11):39334024, 1995.

[12] P Reinicke and J Meyer-ter Vehn. The point explosion with heat conduction. Physics of Fluids A: Fluid Dynamics, 3(7):1807-1818, 1991.

[13] AI Shestakov. Time-dependent simulations of point explosions with heat conduction. Physics of Fluids, 11(5):1091-1095, 1999.

[14] Alan C Calder, Bruce Fryxell, T Plewa, Robert Rosner, LJ Dursi, VG Weirs, T Dupont, HF Robey, JO Kane, BA Remington, et al. On validating an astrophysical simulation code. The Astrophysical Journal Supplement Series, 143(1):201, 2002.

[15] Mark R Krumholz, Richard I Klein, Christopher F McKee, and John Bolstad. Equations and algorithms for mixed-frame flux-limited diffusion radiation hydrodynamics. The Astrophysical Journal, 667(1):626, 2007.

[16] Michael Gittings, Robert Weaver, Michael Clover, Thomas Betlach, Nelson Byrne, Robert Coker, Edward Dendy, Robert Hueckstaedt, Kim New, W Rob Oakes, et al. The rage radiation-hydrodynamic code. Computational Science \& Discovery, 1(1):015005, 2008.

[17] Stephen V Coggeshall and Roy A Axford. Lie group invariance properties of radiation hydrodynamics equations and their associated similarity solutions. The Physics of fluids, 29(8):2398-2420, 1986.

[18] SV Coggeshall. Analytic solutions of hydrodynamics equations. Physics of Fluids A: Fluid Dynamics, 3(5):757-769, 1991.

[19] Robert B Lowrie and Rick M Rauenzahn. Radiative shock solutions in the equilibrium diffusion limit. Shock waves, 16(6):445-453, 2007.

[20] Scott D Ramsey, Emma M Schmidt, Zachary M Boyd, Jennifer F Lilieholm, and Roy S Baty. Converging shock 
flows for a mie-grüneisen equation of state. Physics of Fluids, 30(4):046101, 2018.

[21] Scott D Ramsey and Roy S Baty. Piston driven converging shock waves in a stiffened gas. Physics of Fluids, 31(8):086106, 2019.

[22] Su Bingjing and Gordon L Olson. Benchmark results for the non-equilibrium marshak diffusion problem. Journal of Quantitative Spectroscopy and Radiative Transfer, 56(3):337-351, 1996.

[23] JJ Ruby, JR Rygg, JA Gaffney, B Bachmann, and GW Collins. A boundary condition for guderley's converging shock problem. Physics of Fluids, 31(12):126104, 2019.

[24] Elisha Modelevsky and Re'em Sari. Revisiting the strong shock problem: Converging and diverging shocks in different geometries. arXiv preprint arXiv:2102.07235, 2021.

[25] RE Marshak. Effect of radiation on shock wave behavior. The Physics of Fluids, 1(1):24-29, 1958.

[26] R Pakula and R Sigel. Self-similar expansion of dense matter due to heat transfer by nonlinear conduction. The Physics of fluids, 28(1):232-244, 1985.

[27] N Kaiser, J Meyer-ter Vehn, and R Sigel. The xray-driven heating wave. Physics of Fluids B: Plasma Physics, 1(8):1747-1752, 1989.

[28] James H Hammer and Mordecai D Rosen. A consistent approach to solving the radiation diffusion equation. Physics of Plasmas, 10(5):1829-1845, 2003.

[29] Yves Saillard, Philippe Arnault, and Virginie Silvert. Principles of the radiative ablation modeling. Physics of Plasmas, 17(12):123302, 2010.

[30] Taylor K Lane and Ryan G McClarren. New selfsimilar radiation-hydrodynamics solutions in the highenergy density, equilibrium diffusion limit. New Journal of Physics, 15(9):095013, 2013.

[31] Tomer Shussman and Shay I Heizler. Full self-similar solutions of the subsonic radiative heat equations. Physics of Plasmas, 22(8):082109, 2015.

[32] Shay I Heizler, Tomer Shussman, and Elad Malka. Selfsimilar solution of the subsonic radiative heat equations using a binary equation of state. Journal of Computational and Theoretical Transport, 45(4):256-267, 2016.

[33] Avner P Cohen and Shay I Heizler. Modeling of supersonic radiative marshak waves using simple models and advanced simulations. Journal of Computational and Theoretical Transport, 47(4-6):378-399, 2018.

[34] B Zeldovich Ya and AS Kompaneets. On the propagation of heat for nonlinear heat conduction. Collection dedicated to the seventieth Birthday of Academician AF Ioffe (PI Lukirskii, ed.) Izdat. Acad. Nauk SSSR, Moskow, 1959.

[35] Ya B Zeldovich, Yuri Petrovich Raizer, WD Hayes, and RF Probstein. Physics of shock waves and hightemperature hydrodynamic phenomena. Vol. 2. Academic Press New York, 1967.

[36] Grigory Isaakovich Barenblatt. Scaling, self-similarity, and intermediate asymptotics: dimensional analysis and intermediate asymptotics. Number 14. Cambridge University Press, 1996.

[37] R. E. Pattle. Diffusion from an instantaneous point source with a concentration-dependent coefficient. The Quarterly Journal of Mechanics and Applied Mathematics, 12(4):407-409, 1959.
[38] Ben O'Shaughnessy and Itamar Procaccia. Analytical solutions for diffusion on fractal objects. Physical review letters, 54(5):455, 1985.

[39] Peter Meszaros. Theories of gamma-ray bursts. Annual Review of Astronomy and Astrophysics, 40(1):137-169, 2002.

[40] Jonathan C Tan, Christopher D Matzner, and Christopher F McKee. Trans-relativistic blast waves in supernovae as gamma-ray burst progenitors. The Astrophysical Journal, 551(2):946, 2001.

[41] Nir Sapir, Boaz Katz, and Eli Waxman. Non-relativistic radiation-mediated shock breakouts. i. exact bolometric planar breakout solutions. The Astrophysical Journal, 742(1):36, 2011.

[42] Boaz Katz, Nir Sapir, and Eli Waxman. Non-relativistic radiation mediated shock breakouts. ii. bolometric properties of supernova shock breakout. The Astrophysical Journal, 747(2):147, 2012.

[43] Nir Sapir, Boaz Katz, and Eli Waxman. Non-relativistic radiation mediated shock breakouts. iii. spectral properties of supernova shock breakout. The Astrophysical Journal, 774(1):79, 2013.

[44] NW Evans. The power-law galaxies. Monthly Notices of the Royal Astronomical Society, 267(2):333-360, 1994.

[45] LVE Koopmans, A Bolton, T Treu, O Czoske, MW Auger, M Barnabe, S Vegetti, R Gavazzi, LA Moustakas, and S Burles. The structure and dynamics of massive early-type galaxies: On homology, isothermality, and isotropy inside one effective radius. The Astrophysical Journal Letters, 703(1):L51, 2009.

[46] Peter Schneider and Dominique Sluse. Mass-sheet degeneracy, power-law models and external convergence: Impact on the determination of the hubble constant from gravitational lensing. Astronomy \& Astrophysics, 559:A37, 2013.

[47] Leonid I Sedov. Propagation of strong blast waves. Prikl. Mat. Mekh, 10(2):241-250, 1946.

[48] Geoffrey Ingram Taylor. The formation of a blast wave by a very intense explosion i. theoretical discussion. Proceedings of the Royal Society of London. Series A. Mathematical and Physical Sciences, 201(1065):159-174, 1950.

[49] Leonid Ivanovich Sedov. Similarity and dimensional methods in mechanics. CRC press, 1993.

[50] Eli Waxman and Dov Shvarts. Second-type self-similar solutions to the strong explosion problem. Physics of Fluids A: Fluid Dynamics, 5(4):1035-1046, 1993.

[51] James R Kamm and FX Timmes. On efficient generation of numerically robust sedov solutions. Technical report, Technical Report LA-UR-07-2849, Los Alamos National Laboratory, 2007.

[52] Josselin Garnier, Guy Malinié, Yves Saillard, and Catherine Cherfils-Clérouin. Self-similar solutions for a nonlinear radiation diffusion equation. Physics of plasmas, 13(9):092703, 2006.

[53] Edgar Buckingham. On physically similar systems; illustrations of the use of dimensional equations. Physical review, 4(4):345, 1914.

[54] Almog Yalinewich and Re'em Sari. Analytic asymptotic solution to spherical relativistic shock breakout. Physics of Fluids, 29(1):016103, 2017.

[55] Tamar Faran and Re'em Sari. The non-relativistic interiors of ultra-relativistic explosions: Extension to the blandford-mckee solutions. Physics of Fluids, 33(2):026105, 2021. 
[56] John I Castor. Radiation hydrodynamics. 2004.

[57] Dimitri Mihalas and Barbara Weibel Mihalas. Foundations of radiation hydrodynamics. Courier Corporation, 2013.

[58] Izrail Solomonovich Gradshteyn and Iosif Moiseevich Ryzhik. Table of integrals, series, and products. Academic press, 2014.

\section{Appendix A: Dimensional analysis}

\begin{tabular}{|c|c|c|c|c|}
\hline$w$ & $Q$ & $A$ & $r$ & $t$ \\
\hline \hline$[w]$ & {$[w][\text { length }]^{d-m}$} & $\frac{[\text { length }]^{2-k-m}}{[w]^{n}[\text { time }]}$ & {$[$ length $]$} & {$[$ time $]$} \\
\hline
\end{tabular}

Table III. The dimensional quantities in the problem (upper line) and their dimensions (lower line).

In this appendix we will use the method of dimensional analysis in order to find a self-similar ansatz for the solution of the problem defined by equations $(17)$ and $(19)$. The dimensional quantities which define the problem are listed in table III It is seen that the problem is defined by $M=5$ dimensional quantities which are composed of $N=3$ different units. Therefore from the central theorem of dimensional analysis (a.k.a. the Pi theorem) [35. 36. 53, the problem can be solved using $M-N=2$ dimensionless variables, written in terms of power laws of the dimensional quantities:

$$
\begin{gathered}
\xi=r Q^{x} A^{y} t^{z}, \\
f(\xi)=\frac{w}{Q^{a} A^{b} t^{c}} .
\end{gathered}
$$

The requirement that $\xi$ is dimensionless gives:

$$
\begin{aligned}
& x-y n=0 \\
& 1+(d-m) x+(2-k-m) y=0 \\
& -y+z=0
\end{aligned}
$$

which has the solution:

$$
\begin{gathered}
x=-\frac{n}{p}, \\
y=z=-\frac{1}{p},
\end{gathered}
$$

where $p$ is given by Eq. (24). Similarly, the requirement that $f(\xi)$ is dimensionless gives:

$$
\begin{aligned}
& 1-a+b n=0 \\
& -a(d-m)-(2-k-m) b=0 \\
& b-c=0
\end{aligned}
$$

which has the solution:

$$
\begin{gathered}
a=\frac{2-k-m}{p}, \\
b=c=-\frac{d-m}{p} .
\end{gathered}
$$

Hence, it is seen that the resulting dimensionless quantities (A1)- A2 give the self-similar ansatz 222)-(23). 


\section{Appendix B: The calculation of $\xi_{0}$}

The value of $\xi_{0}$ can be found by substituting Eq. 42 into the energy conservation constraint 29. This substitution results in the following equation:

$$
\frac{n^{\frac{1}{n}} \mathcal{A}_{d} \xi_{0}^{\frac{2-k-m}{n}}+d-m}{(p|2-k-m|)^{\frac{1}{n}}} I=1,
$$

where $I$ is the following definite integral:

$$
I=\int_{0}^{1} x^{d-m-1}\left|1-x^{2-k-m}\right|^{\frac{1}{n}} d x .
$$

Under the conditions $2-k-m>0, d-m>0$ and $\frac{1}{n}+1>0$, this integral is well known (i.e. Ref. [58], page 324, Eq. 3.251-1), and given by:

$$
I=\frac{1}{2-k-m} \mathcal{B}\left(\frac{d-m}{2-k-m}, \frac{1}{n}+1\right),
$$

with the Beta function defined in terms of the Gamma function:

$$
\mathcal{B}(x, y)=\frac{\Gamma(x) \Gamma(y)}{\Gamma(x+y)} .
$$

On the other hand, if $2-k-m<0$, we employ the integral (see i.e. Ref. [58] page 325, Eq. 3.251-3):

$$
\int_{1}^{\infty} x^{s-1}\left(x^{q}-1\right)^{v-1} d x=\frac{1}{q} \mathcal{B}\left(1-v-\frac{s}{q}, v\right),
$$

which is valid for $q>0, v>0$, and $s<q(1-v)$, so that after a change of variables $y=\frac{1}{x}$ in Eq. B2, one finds that:

$$
I=-\frac{1}{2-k-m} \mathcal{B}\left(-\frac{1}{n}-\frac{d-m}{2-k-m}, \frac{1}{n}+1\right),
$$

which is valid under the condition $\frac{1}{n}+1>0$ and $2-k-$ $m+(d-m) n>0$, which is already assumed in Eq. (34). Using the results in equations $\sqrt{\mathrm{B} 3}),(\mathrm{B} 4)$ in Eq. (B) and solving for $\xi_{0}$, results in equations $(44)-45$.

\section{Appendix C: Analytic solution for $\omega=\omega_{c}$}

For $\omega=\omega_{c}($ for which $2-k-m=0)$, the ODE 28 reads:

$$
n f^{n-1}(\xi) f^{\prime}(\xi)=-\frac{1}{(d-m) \xi}
$$

Assuming $n>0$, and employing the boundary condition at infinity (see Eq. (30), gives the solution:

$$
f(\xi)= \begin{cases}{\left[\frac{\ln \left(\frac{\xi_{0}}{\xi}\right)}{d-m}\right]^{\frac{1}{n}},} & \xi<\xi_{0} \\ 0, & \xi>\xi_{0}\end{cases}
$$

where $\xi_{0}$ is a constant of integration, which can be obtained, as done in Appendix ( $\sqrt{\mathrm{B}}$, by employing the energy conservation constraint 29 . This gives:

$$
\frac{\mathcal{A}_{d} \xi_{0}^{d-m}}{(d-m)^{\frac{1}{n}}} I=1
$$

where $I$ is the following definite integral:

$$
I=\int_{0}^{1}\left[\ln \left(\frac{1}{x}\right)\right]^{\frac{1}{n}} x^{d-m-1} d x=\frac{\Gamma\left(1+\frac{1}{n}\right)}{(d-m)^{1+\frac{1}{n}}}
$$

where we have used a well known integral identity (i.e. Ref. 58] page 551, Eq. 4.272-6), which is valid under the condition $d-m>0$ (which must hold for $\omega=\omega_{c}$, since we assume Eq. (34)). Using Eq. (C2) in Eq. (C1) and solving for $\xi_{0}$, results in Eq. 50 . 